\title{
Outcome of adenotonsillectomy in children with Down syndrome and obstructive sleep apnoea
}

\author{
Mieke Maris, ${ }^{1}$ Stijn Verhulst, ${ }^{2}$ Marek Wojciechowski, ${ }^{2}$ Paul Van de Heyning, ${ }^{1}$ \\ An Boudewyns ${ }^{1}$
}

'Department of

Otorhinolaryngology, Head and Neck Surgery, Antwerp University Hospital, University of Antwerp, Antwerp, Belgium ${ }^{2}$ Department of Pediatrics, Antwerp University Hospital, University of Antwerp, Antwerp, Belgium

\section{Correspondence to}

Professor An Boudewyns, Antwerp University Hospital, Wilrijkstraat 10, Edegem (Antwerp) B-2650, Belgium; an. boudewyns@uza.be

Received 15 December 2015 Revised 14 June 2016 Accepted 10 July 2016 Published Online First 2 August 2016

\section{CrossMark}

To cite: Maris M, Verhulst $S$, Wojciechowski M, et al. Arch Dis Child 2017;102:

331-336.
ABSTRACT

Objective To evaluate the outcome of adenotonsillectomy (AT) in a cohort of children with Down syndrome (DS) and obstructive sleep apnoea (OSA).

Design Retrospective, cross-sectional study.

Setting Tertiary care centre.

Patients Children with DS and OSA, without previous upper airway (UA) surgery.

Interventions AT and full overnight polysomnography. Main outcome results A significant improvement of the obstructive apnoea-hypopnoea index (oAHI) after AT was obtained. No differences in sleep efficiency or sleep fragmentation were found postoperatively. Almost half of the children had persistent OSA (OAHI $\geq 5 /$ hour).

Results Data are presented as median (lower-upper quartile). Thirty-four children were included, median age 4.0 years $(2.7-5.8)$, body mass index (BMI) $z$-score 0.81 $(-0.46-1.76)$, and oAHI 11.4/hour (6.5-22.7). The majority presented with severe OSA (58.9\%). AT was performed in 22 children, tonsillectomy in 10 and adenoidectomy in two. Postoperatively, a significant improvement of the $\mathrm{OAHI}$ was measured from 11.4 /hour $(6.5-22.7)$ to $3.6 /$ hour $(2.1-9.5)(p=0.001)$, with a parallel increase of the minimum oxygen saturation $(p=0.008)$. Children with initially more severe OSA had significantly more improvement after UA surgery $(p=0.001)$. Persistent OSA was found in $47.1 \%$ of the children.

Conclusions AT results in a significant improvement of OSA in children with DS without a change in sleep efficiency or sleep stage distribution. Severe OSA was associated with a larger reduction of OSA severity. Almost half of the children had persistent OSA, which was not correlated to age, gender or BMI z-score.

\section{INTRODUCTION}

Children with Down syndrome (DS) are increasingly diagnosed with sleep disordered breathing, including obstructive sleep apnoea (OSA). The prevalence of OSA in children with DS is estimated at between $30-60 \%$, compared to $0.7-2.0 \%$ in a general paediatric population. ${ }^{1-3}$ In the great majority of paediatric patients, OSA is caused by adenotonsillar hypertrophy. Children with DS present with multiple anatomical and functional factors contributing to the development of OSA. This includes midfacial hypoplasia, a narrow nasopharynx, a small oropharynx, relative macroglossia and adenotonsillar hypertrophy, more respiratory infections (and more secretions) due to an immature immune system, general hypotonia, obesity, and gastro-oesophageal reflux disease. ${ }^{1}{ }^{4}$ The

\section{What is already known on this topic?}

- Children with Down syndrome (DS) have a high prevalence of obstructive sleep apnoea (OSA).

- Small studies, with methodological differences, showed a significant improvement after upper airway surgery.

- There is a high prevalence of persistent OSA.

\section{What this study adds?}

- Outcome of adenotonsillectomy (AT) was evaluated by pre- and postoperative polysomnography in a large homogeneous study population of young children with DS, without previous upper airway surgery.

- A significant improvement in OSA severity was found after AT.

- Children with more severe OSA showed a larger improvement in OSA severity after AT.

- Persistent OSA was present in almost half of the DS children and this could not be predicted by patient related factors.

negative health impact of OSA on neurocognitive development and behaviour is previously well described. $^{5} 6$ Recognition, diagnosis, and subsequent treatment of sleep-disordered breathing will be mandatory to provide adequate healthcare in patients with DS.

Adenotonsillectomy (AT) is the first line treatment for paediatric OSA; however, previous studies have reported that this treatment is less curative in children with DS. The prevalence of persistent OSA in children with DS varies between $30-50 \%,{ }^{7}$ compared to $20-25 \%$ in otherwise healthy children. ${ }^{8}$ High prevalence rates of persistent OSA are reported in the literature, although some studies report a significant improvement in OSA severity (obstructive apnoea-hypopnoea index (oAHI)) after AT in children with DS. ${ }^{10}$ However, previous studies evaluating the outcome of AT in children with DS and OSA have only included small study populations, and only a few studies objectively documented outcome results with pre- and postoperative polysomnography (PSG) data. ${ }^{9} 11$

The first aim of this study was to evaluate the outcome of AT in children with DS and OSA, documented with pre- and postoperative PSG data. 
Secondly, we evaluated which patient-related factors are associated with persistent OSA.

\section{METHODS}

We retrospectively analysed the data of all children enrolled in the multidisciplinary Down Team (Antwerp, University Hospital Antwerp, Belgium) and seen at the ear nose and throat (ENT) department between January 2010 and September 2015. Children with a PSG-confirmed diagnosis without previous upper airway (UA) surgery were eligible for inclusion. A clinical examination was performed in every child and tonsil size was scored according to the Brodsky score. ${ }^{12}$ Body mass index (BMI) z-scores were calculated according to Flemish growth curves for boys and girls.

All children had a full overnight PSG, where the following variables were continuously measured and recorded by a computerised PSG (brain RT, OSG, Rumst, Belgium): electroencephalography (central leads: C4 and C3, frontal leads: F3 and F4); electro-oculography; electromyography of anterior tibialis and chin muscles; and electrocardiography. Respiratory effort was measured by respiratory inductance plethysmography and oxygen saturation by a finger probe connected to a pulse oximeter. Airflow was measured by means of a nasal pressure cannula and thermistor, and snoring was detected by means of a microphone at the suprasternal notch. Children were also monitored on audio/videotape using an infrared camera. Polysomnograms were manually scored by certified technicians according to the American Academy of Sleep Medicine (AASM) guidelines for children. ${ }^{13} \mathrm{~A}$ diagnosis of OSA is established with an oAHI $\geq 2$ /hour. ${ }^{14}$ OSA severity was defined as mild with an oAHI $>2$ /hour and $<5$ /hour, moderate with an oAHI $\geq 5$ /hour and $<10$ /hour, and severe OSA with an oAHI $\geq 10 /$ hour.

Children with moderate or severe OSA were eligible for AT. Surgery was proposed in children with mild OSA after failure of medical treatment (nasal corticosteroids and/or montelukast, used at least for 6 months) or upon specific clinical indications. Surgery was performed under general anaesthesia with cold instruments. In case of tonsillectomy, anterior and posterior pillars were sutured with Vycril 3.0 resorbable sutures. No intraor subcapsular technique was used in any patient. All children were admitted to the paediatric ward for pulse oximetry and monitoring, and stayed overnight for at least 1 day. A PSG was scheduled at 3 months postoperatively. Delta oAHI ( $\triangle$ oAHI) is calculated as the difference between preoperative and postoperative oAHI. Persistent OSA is defined as an oAHI $\geq 5$ / hour. $^{8} 15$

During the study period, 125 children with DS had a full overnight PSG. In 90 of these children a diagnosis of OSA was established (72.0\%), with an oAHI 8.7/hour (4.1-16.7). The majority of the children presented with severe OSA $(43.8 \%)$, $24.7 \%$ with moderate OSA, and $31.5 \%$ with mild OSA. The treatment decision for this group was based upon OSA severity, clinical findings, parent's preferences, and information about the pattern of UA obstruction obtained during drug-induced sedation endoscopy. AT was performed in 39 children (including seven children with mild OSA after failure of medical treatment). The remainder were not considered to be suitable candidates for AT based upon findings during drug-induced sedation endoscopy and these children were treated by continuous positive airway pressure (CPAP) $(n=9)$, orthodontics $(n=3)$, proton pump inhibitors $(n=1)$, montelukast and/or nasal corticosteroids $(n=4)$, a sleep positioner trainer $(n=4)$ or lingual tonsillectomy $(n=1)$. Pre- and postoperative PSG data were available for 34 children (in one child registration failed due to the lack of cooperation, three children were lost in follow-up, and one child did not show up at the time of the postoperative PSG, and a new PSG was refused by the parents). These 34 surgically treated children (20 children with severe OSA, seven with moderate OSA, and seven with mild OSA) for whom pre-and postoperative data are available, constitute the study population for the present paper. This study was approved by the local ethics committee.

Data are presented as median values and lower and upper quartile. Non-parametric statistical analysis was performed with IBM SPSS V.22.0 since data were not normally distributed. Pre-and postoperative data were compared with a Wilcoxon signed rank test. Patient related factors for persistent OSA were evaluated with a Mann-Whitney U test, Spearman correlation analysis for continuous variables, and $\chi^{2}$ test for categorical variables. Level of significance was set at a value of $\mathrm{p}<0.05$.

\section{RESULTS}

The study population consists of 34 young, non-obese DS children; age 4.0 years (2.7-5.8), BMI z-score $0.81(-0.46-1.76)$ with severe OSA (oAHI 11.4/hour (6.5-22.7)). Twenty children $(58.9 \%)$ presented with severe OSA, seven $(20.6 \%)$ with moderate OSA, and seven $(20.6 \%)$ with mild OSA. Half of the children were boys, and almost half $(41.2 \%)$ had one or more comorbidities (underlying cardiac disease and/or hypothyroidism). Children with comorbidity presented with significantly more severe OSA (oAHI) $(p=0.020)$.

Most of the children underwent an AT $(n=22)$. Ten children underwent an isolated tonsillectomy $(\mathrm{n}=10)$ and only two were treated by adenoidectomy $(n=2)$, both based upon findings from drug-induced sedation endoscopy. Postoperative PSG was performed after 3.6 months (3.1-4.7). Pre- and postoperative patient characteristics and polysomnographic data for the whole study group are presented in table 1 .

Upper airway surgery ((A)T) resulted in a significant decrease in oAHI as presented in figure 1 , associated with a significant increase in minimum oxygen saturation $\left(\mathrm{MinSatO}_{2}\right)$. There was no change in mean oxygen saturation $\left(\mathrm{MeanSatO} \mathrm{O}_{2}\right)$, sleep efficiency, arousal index or sleep stage distribution. Postoperative PSG parameters were compared between those children who

Table 1 Pre- and postoperative patient characteristics and PSG outcome data

\begin{tabular}{|c|c|c|c|}
\hline Variable & Preoperative value & Postoperative value & p Value \\
\hline Age (years) & $4.0(2.7-5.8)$ & $4.7(3.1-6.5)$ & $0.001^{*}$ \\
\hline BMI z-score & $0.8(-0.5-1.8)$ & $0.9(0.3-1.4)$ & 0.332 \\
\hline oAHI (/hour) & $11.4(6.5-22.7)$ & $3.6(2.1-9.5)$ & 0.001 * \\
\hline MeanSatO $_{2}(\%)$ & $96.3(95.0-96.9)$ & $96.6(95.2-97.2)$ & 0.254 \\
\hline $\mathrm{MinSatO}_{2}(\%)$ & $84.0(79.5-89.0)$ & $88.5(84.0-90.1)$ & $0.008^{*}$ \\
\hline ODI (n/hour) & $5.6(1.8-12.6)$ & $2.9(1.1-5.8)$ & 0.093 \\
\hline Sleep efficiency (\%) & $86.0(78.5-91.4)$ & $88.8(82.9-92.1)$ & 0.207 \\
\hline Arousal index (n) & $9.5(0.6-20.2)$ & $8.6(1.5-14.9)$ & 0.143 \\
\hline Stage 1 (\% TST) & $1.6(1.0-3.0)$ & $2.0(1.0-4.1)$ & 0.567 \\
\hline Stage 2 (\% TST) & $44.4(33.7-49.0)$ & $45.7(37.0-48.9)$ & 0.654 \\
\hline Stage 3 (\% TST) & $24.1(17.3-29.5)$ & $20.7(17.7-25.5)$ & 0.304 \\
\hline REM sleep (\% TST) & $25.6(20.2-32.4)$ & $27.7(23.0-32.0)$ & 0.194 \\
\hline
\end{tabular}


underwent tonsillectomy and AT as presented in table 2. Since only two children were treated by adenoidectomy, this number is too small for statistical analysis. A significant improvement in oAHI $(\mathrm{p}=0.001), \mathrm{MinSatO}_{2}(\mathrm{p}=0.020)$, and oxygen desaturation index (ODI) $(p=0.034)$ was obtained in those children treated by AT.

Sixteen children (47.1\%) had persistent OSA (oAHI $\geq 5 /$ hour) with a postoperative oAHI of 11.1 /hour (6.8-17.2). Only six children $(17.6 \%)$ had a normal oAHI $(<2 /$ hour) after (A)T. Percentages of children according to OSA severity pre-and postoperatively are presented in figure 2. Eight children presented with a higher postoperative oAHI. Statistical analysis did not show any contribution of specific risk factors (no significant postoperative weight gain) or other comorbidities such a thyroid dysfunction, cardiac disease or asthma. Three children had a common cold, but no fever, during control PSG.

There was no association between preoperative tonsil score, age or gender with OSA severity (oAHI). A significant correlation was found between BMI z-score and OSA severity (oAHI). Persistent OSA could not be predicted by the preoperative findings such as BMI z-score, gender, age or oAHI or the presence of comorbidities (cardiac disease or hypothyroidism). These results are summarised in table 3. Eight children presented with an increased oAHI postoperatively. These children did not show

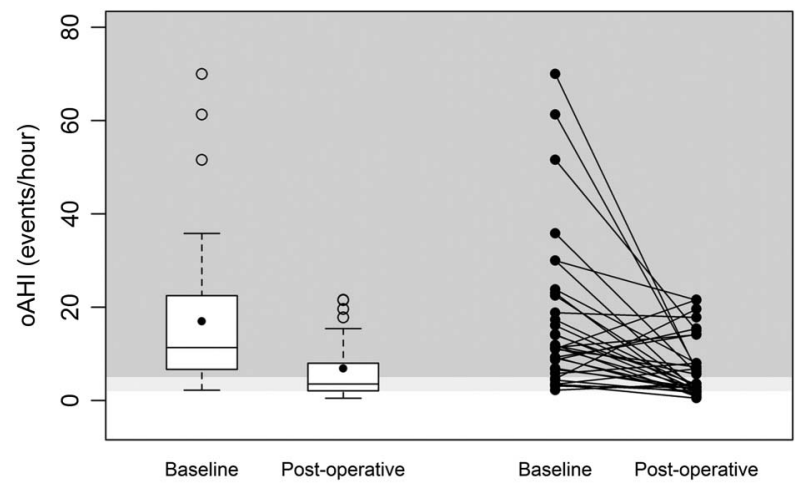

Figure 1 Pre- and postoperative obstructive apnoea-hypopnoea index (oAHI) values in 34 patients with Down syndrome. Left-hand plot shows median values with upper and lower quartile. Right hand plot shows individual values. any specific risk factor (no significant postoperative weight gain) or other comorbidities such as thyroid dysfunction, cardiac disease or asthma. Children with more severe OSA at baseline had a greater reduction in oAHI $(\Delta \mathrm{oAHI})(\mathrm{p}=0.001, \mathrm{r}=-0.8)$, as depicted in figure 3.

Four children $(11.8 \%)$ encountered postoperative problems during the first night or week. A one-and-a-half-year-old child presented with respiratory problems during the first postoperative night, and this child was admitted to the intensive paediatric care unit during one night. This child had a history of bronchiolitis at a younger age. Three other children presented with fever in the first postoperative week. One of them was readmitted to the hospital because of insufficient oral intake. Intravenous fluid therapy was started and the child recovered completely. One child presented with rebleeding after tonsillectomy and a revision surgery was performed the first postoperative day.

\section{DISCUSSION}

To the best of our knowledge, this is the first study including more than 30 children with DS and OSA $(n=34)$, for whom pre-and postoperative PSG data are available.

We found a significant improvement of OSA (reduction of oAHI, improvement in $\mathrm{MinSatO}_{2}$ and ODI) after AT, but almost half of the patients still showed persistent disease. Tonsillectomy did not result in a significant improvement of respiratory parameters. This might be related to the small number of children $(n=10)$ undergoing tonsillectomy. The number of children treated by adenoidectomy $(n=2)$ is too small to allow for meaningful statistical analysis. Our present data are in accordance with previous reports on outcome of UA surgery for children with DS. A recent publication by Thottam et al reports a significant improvement of the oAHI after AT in 18 children with DS (oAHI from 26.6/hour preoperatively to 11.6/hour postoperatively). In their study only children with severe OSA were included, and the percentage of children with persistent OSA is not reported. ${ }^{16}$ Merrell and Shott reported a normal apnoeahypopnoea index (AHI), defined as an AHI $<1$ /hour, in $52 \%$ after AT in 16 patients with DS, aged 3.7 years (2.3-5.7). ${ }^{11}$ Compared to our study population, they included a smaller study group who presented with mild OSA (AHI 6.3/hour). Shete et $a l^{9}$ also found a significant reduction in oAHI postoperatively, and only $18 \%$ reached a normal AHI

Table 2 Pre- and postoperative comparison of PSG data for children treated by adenotonsillectomy and tonsillectomy

\begin{tabular}{|c|c|c|c|c|}
\hline \multirow[b]{2}{*}{ Variable } & \multicolumn{2}{|c|}{ Adenotonsillectomy ( $n=22$ ) } & \multicolumn{2}{|c|}{ Tonsillectomy $(n=10)$} \\
\hline & Preoperative & Postoperative & Preoperative & Postoperative \\
\hline oAHI (n/hour) & $11.9(6.2-19.9)$ & $8.0(2.0-6.9)^{*}$ & $9.1(5.1-24.4)$ & $5.2(2.6-16.9)$ \\
\hline MeanSatO $\mathrm{O}_{2}(\%)$ & $96.2(95.0-96.7)$ & $96.3(95.0-97.3)$ & $96.7(95.8-97.3)$ & $96.8(95.8-97.1)$ \\
\hline $\mathrm{MinSatO}_{2}(\%)$ & $83.1(77.0-88.5)$ & $88.0(84.0-90.0)^{* *}$ & $88.0(86.0-94.0)$ & $89.0(86.0-94.0)$ \\
\hline ODI (n/hour) & $5.8(2.6-12.6)$ & $2.9(1.1-6.5)^{\star * *}$ & $3.4(0.5-16.8)$ & $2.9(0.8-4.5)$ \\
\hline SE $(\%)$ & $86.0(77.2-90.0)$ & $88.6(82.9-91.9)$ & $84.6(78.0-92.0)$ & $89.8(83.5-92.5)$ \\
\hline Al (n/hour) & $17.7(0.6-30.9)$ & $8.9(0.7-17.1)$ & $6.7(1.0-9.5)$ & $6.7(1.0-11.0)$ \\
\hline REM (\%TST) & $25.9(22.3-35.8)$ & $28.2(22.3-31.0)$ & $20.6(15.0-27.3)$ & $26.0(21.2-33.5)$ \\
\hline S1 (\%TST) & $1.6(0.9-2.2)$ & $2.0(0.8-4.1)$ & $2.9(0.8-8.2)$ & $2.9(1.0-11.5)$ \\
\hline S2 (\%TST) & $45.1(38.1-49.0)$ & $45.8(37.5-48.9)$ & $40.5(21.4-57.5)$ & $45.7(37.0-50.2)$ \\
\hline S3 (\%TST) & $24.1(16.2-27.2)$ & $22.9(19.2-26.0)$ & $22.5(16.0-39.0)$ & $18.0(16.9-21.5)$ \\
\hline
\end{tabular}

Statistical analysis: ${ }^{*} \mathrm{p}=0.001 ;{ }^{* *} \mathrm{p}=0.02 ;{ }^{* * *} \mathrm{p}=0.034$

$\mathrm{Al}$, apnoea index; $\mathrm{MeanSatO}_{2}$, mean oxygen saturation; $\mathrm{MinSat}_{2}$, minimum oxygen saturation; oAHI, obstructive apnoea-hypopnoea index; ODI, oxygen desaturation index; PSG, polysomnography; REM, rapid eye movement; SE, sleep efficiency; TST, total sleep time. 
postoperatively (defined as an AHI $<2 /$ hour). These figures are similar to our present findings where $17.6 \%$ of the children obtained an oAHI $<2$ /hour. Shete et al did not report on the severity of persistent OSA but $55 \%$ of the patients in that study were treated by CPAP postoperatively. Their study differs in other aspects as well: they included only 11 patients with DS, and the majority of the children were obese and they were older (mean age 8.5 years) than our study population. ${ }^{9}$ In our study seven children with preoperative mild OSA were included. Two of these children presented with a postoperative oAHI between 2 and 5/hour. These children were considered to be successfully treated (oAHI $>5 /$ hour), although not completely cured (oAHI $<2$ /hour). If these children with preoperative mild OSA were excluded for statistical analysis, and only those children with moderate to severe OSA were included, similar treatment outcomes were found with persistent disease in $48.1 \%$ and a complete cure in $18.5 \%$.

Despite a significant improvement in OSA severity found after (A)T, the high prevalence of persistent OSA in children with DS poses a challenge for further treatment. Several authors recommend an evaluation of the UA to identify the level(s) of UA collapse in children with persistent OSA after AT. ${ }^{17} 18$ Donnelly et $a l^{19}$ performed cine-MRI in 27 DS patients with persistent OSA after AT and attributed the persistence of OSA to multiple causes such as macroglossia, glossoptosis, recurrent enlargement of the adenoids, and enlargement of the lingual tonsils. Additional treatment may be needed to address these levels of UA obstruction in order to achieve a complete cure.

Some authors propose more aggressive surgical procedures in children with DS and OSA because of the rather low success rate of (A)T. However, no objective outcome measurements were used in most of these previous studies. Tang et $a l^{20}$ recently reported gaps in evidence in the management of OSA

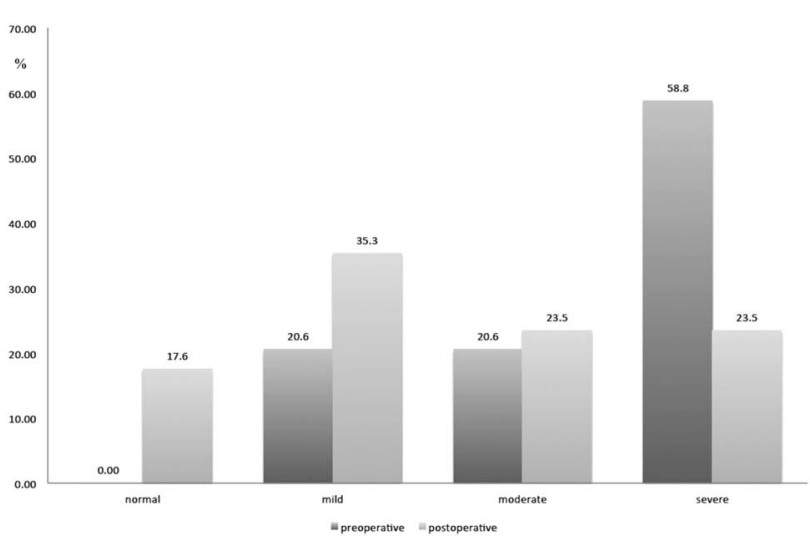

Figure 2 Percentage of children in each category of obstructive sleep apnoea severity, pre- and postoperatively. in children with DS, including the appropriate use of PSG and effectiveness of surgical procedures performed alone or in combination. Some authors recommend a combined surgical approach, including soft tissue surgery and facial skeletal surgery, since only limited success is obtained with soft tissue surgery. The main goal is to avoid tracheostomy in these children with persistent OSA. Lefaivre et al published the results of more aggressive multilevel combined surgery (uvulopalatopharyngoplasty (UPPP), tongue reduction, tongue hyoid advancement, midface advancement) in seven patients with DS, documented with pre- and postoperative PSG data in six patients. All had a symptomatic improvement, but half of the studied patients presented initially with mild OSA resulting in an $\mathrm{OAHI}<5 /$ hour postoperatively in all subjects, and a complete cure with an oAHI $<2 /$ hour in four patients. ${ }^{21}$ Complications of these combined surgical techniques are frequent, and longterm outcomes are unknown. ${ }^{22}$ de Moura et $a l^{23}$ reported an improvement of parental perception of symptoms of sleepdisordered breathing after rapid maxillary expansion, but in this study objective postoperative data are also lacking. Besides surgical treatment, non-surgical management of paediatric OSA should be considered, especially when surgical interventions carry a high risk for the patient (due to comorbidities). Non-surgical treatment options are CPAP, orthodontics or medical treatment. ${ }^{24}$

In conclusion, there are only a few publications reporting objective outcome data of extensive surgical treatment modalities in children with DS. Great care should be taken in selecting patients, and parents should be aware of the possible complications of different treatment modalities, and the higher risk of persistent OSA compared to normal developing children.

Persistent OSA could not be predicted by preoperative BMI $\mathrm{z}$-score, age, gender or baseline oAHI. In a recent study higher age, respiratory distress index and BMI emerged as significant risk factors for unfavourable treatment outcome after (A) $\mathrm{T}^{25}$ and several studies reported an association of obesity with persistent OSA. ${ }^{26} 27$ This could not be confirmed in our study. Although children with DS are susceptible to obesity, our study population was generally not obese, which might partially explain the lack of an association between persistent OSA and BMI z-score. Only a minority of the present study population presented with obesity (17.6\%). This might be explained by the rather young study population and the fact that all DS children at our institution are enrolled in a weight control programme that might result in a selection bias towards non-obese DS children to be included. Moreover, different criteria of obesity are used in the literature. Tagaya et $a l^{28}$ defined childhood obesity as a BMI z-score $>2$. Bhattacharjee et $a l^{15}$ considered a BMI z-score $>1.65$ as an indicator of obesity. Corresponding with this definition, $29.4 \%$ of this study population was considered obese. Although no correlation was found between BMI z-score

Table 3 Results of correlation analysis between OSA severity (oAHI), persistent OSA and patient characteristics (BMI z-score, age, gender, tonsil score) and preoperative oAHI

\begin{tabular}{|c|c|c|c|c|c|}
\hline & BMI z-score & Age (years) & Gender & Tonsil score & Preoperative oAHI (n/hour) \\
\hline OSA severity (oAHI) & $0.4(0.034)^{*}$ & $0.2(0.387)$ & $-0.4(0.692)$ & $-0.2(0.275)$ & - \\
\hline $\begin{array}{l}\text { Persistent OSA } \\
\text { (oAHI } \geq 5 / \text { hour) }\end{array}$ & $-0.6(0.569)$ & $-1.1(0.277)$ & $0.5(0.492)$ & - & $-1.6(0.120)$ \\
\hline
\end{tabular}




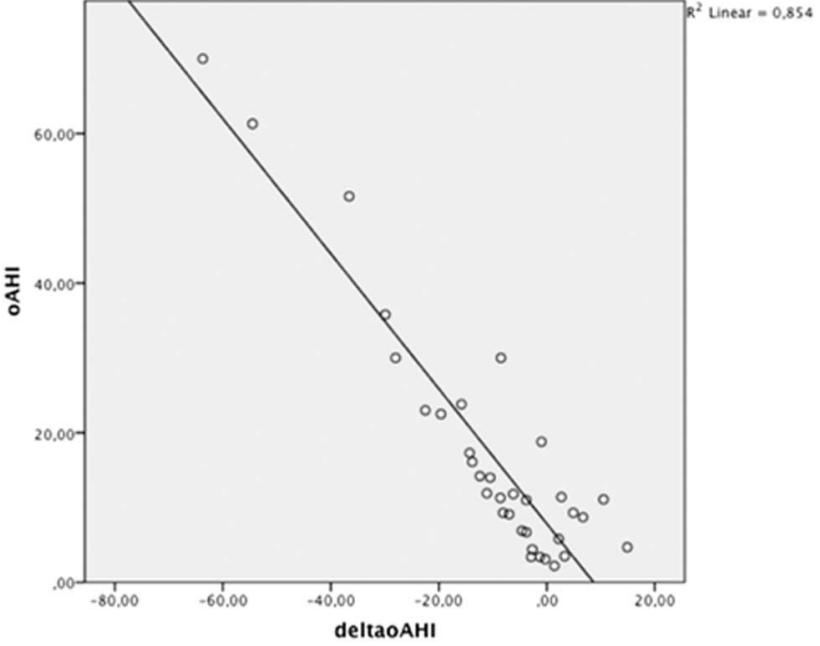

Figure 3 Scatterplot correlation oAHIpreop (oAHI preoperative) and delta $\mathrm{OAHI}$ (reduction in $\mathrm{OAHI}$ postoperative). $\mathrm{OAHI}$, obstructive apnoea-hypopnoea index.

and obesity, children with an increased BMI z-score presented with more severe OSA (higher oAHI).

We did not find a change in sleep architecture after (A)T. This observation is also in line with the data of Shete et $a l^{9}$ where no significant effect was seen on the different sleep parameters post-AT. They reported similar findings in children with DS postoperatively. Levanon $e t a l^{29}$ reported more sleep fragmentation in children with DS, which was only partially related to underlying OSA, compared to normal developing children. Nisbet et $a l^{30}$ confirmed this altered sleep architecture in children with DS. Previous studies showed an improvement in oAHI and sleep efficiency after AT, without altering sleep architecture in normal developing children. ${ }^{8}{ }^{30}$ However, a recent meta-analysis showed a notable improvement in respiratory parameters after AT, along with a reduction in stage 1 sleep, and an increase in slow wave sleep and rapid eye movement sleep, especially in non-obese individuals. ${ }^{26}$

In this study, $11.8 \%$ of the patients presented with postoperative problems, which is less compared to other reports. Goldstein et $a l^{31}$ and Bower et $a l^{32}$ report respiratory complications in up to $25 \%$ of the patients. Also Thottam et al ${ }^{16}$ reported significantly more postoperative problems in children with DS after AT and a prolonged hospitalisation compared to normal developing children. Differences in OSA severity, obesity and comorbid conditions such as congenital heart disease might explain these differing figures. We nevertheless strongly support the recommendation that children with DS should be admitted to the hospital overnight after UA surgery because of the higher risk of respiratory complications due to underlying predisposing factors.

\section{CONCLUSION}

AT results in a significant improvement in OSA severity in children with DS, without a change in sleep efficiency or sleep architecture. Almost half of the children had persistent OSA. Severe OSA was associated with a larger improvement in OSA severity and there was no correlation between gender, BMI z-score, age and persistent OSA. Further treatment of persistent OSA in children with DS remains a challenge, and a multidisciplinary approach is mandatory using both subjective and objective outcome measurements.
Funding Research Foundation Flanders (Senior Clinical Investigator grant/ $1842113 \mathrm{~N})$

Competing interests None declared.

Ethics approval Institutional Review Board of the Antwerp University Hospital, BE 2650 Antwerp.

Provenance and peer review Not commissioned; externally peer reviewed.

\section{REFERENCES}

1 Shott SR. Down syndrome: common otolaryngologic manifestations. AM J Med Genet C Semin Med Genet 2006;142C:131-40.

2 Maris $M$, Verhulst $S$, Wojciechowski $M$, et al. Prevalence of obstructive sleep apnea in children with Down syndrome. Sleep 2016;39:699-704.

$3 \mathrm{Ng} \mathrm{DK}$, Hui HN, Chan $\mathrm{CH}$, et al. Obstructive sleep apnoea in children with Down syndrome. Sing M J 2006;47:774-9.

4 Mitchell RB, Call E, Kelly J. Ear, nose and throat disorders in children with Down syndrome. Laryngoscope 2003;113:259-63.

5 Breslin J, Spanò G, Bootzin R, et al. Obstructive sleep apnea syndrome and cognition in Down syndrome. Dev Med Child Neurol 2014;56:657-64.

6 Shott SR, Amin R, Chini B, et al. Obstructive sleep apnea: should all children with Down syndrome be tested? Arch Otolaryngol Head Neck Surg 2006;132:432-6.

7 Shott SR, Donnelly LF. Cine magnetic resonance imaging: evaluation of persistent airway obstruction after tonsil and adenoidectomy in children with Down syndrome. Laryngoscope 2004;114:1724-9.

8 Mitchell RB. Adenotonsillectomy for obstructive sleep apnea in children: outcome evaluated by pre- and postoperative polysomnography. Laryngoscope 2007; 117:1844-54.

9 Shete MM, Stocks RM, Sebelik ME, et al. Effects of adeno-tonsillectomy on polysomnography patterns in Down syndrome children with obstructive sleep apnea: a comparative study with children without Down syndrome. Int J Ped Otorhinolaryngol 2010;74:241-4.

10 Thottam PJ, Choi S, Simons JP, et al. Effect of adenotonsillectomy on central and obstructive sleep apnea in children with Down syndrome. Otolaryngol Head Neck Surg 2015;153:644-8.

11 Merrell JA, Shott SR. OSAS in Down syndrome: T\&A versus T\&A plus lateral pharyngoplasty. Int J Ped Otorhinolaryngol 2007;71:1197-203.

12 Brodsky L. Modern assessment of tonsils and adenoids. Ped Clin North Am 1989;36:1551-69.

13 Berry RB, Budhiraja R, Gottlieb DJ, et al. Rules for scoring respiratory events in sleep: update of the 2007 AASM Manual for the Scoring of Sleep and Associated Events. Deliberations of the Sleep Apnea Definitions Task Force of the American Academy of Sleep Medicine. J Clin Sleep Med 2012;8:597-619.

14 Marcus $\mathrm{CL}$, Moore $\mathrm{RH}$, Rosen $\mathrm{CL}$, et al. A randomized trial of adenotonsillectomy for childhood sleep apnea. N Eng J Med 2013;368:2366-76.

15 Bhattacharjee R, Kheirandish-Gozal L, Spruyt K, et al. Adenotonsillectomy outcomes in treatment of obstructive sleep apnea in children: a multicenter retrospective study. Am J Respir Crit Care Med 2010;182:676-83.

16 Thottam PJ, Trivedi S, Siegel B, et al. Comparative outcomes of severe obstructive sleep apnea in pediatric patients with trisomy 21. Int J Ped Otorhinolaryngol 2015:79:1013-16.

17 Fung $\mathrm{E}$, Witmans $\mathrm{M}$, Ghosh $\mathrm{M}$, et al. Upper airway findings in children with Down syndrome on sleep nasopharyngoscopy: case-control study. J Otolaryngol Head Neck Surg 2012;41:138-44.

18 Durr ML, Meyer AK, Kezirian EJ, et al. Drug-induced sleep endoscopy in persistent pediatric sleep-disordered breathing after adenotonsillectomy. Arch Otolaryngol Head Neck Surg 2012;138:638-43.

19 Donnelly LF, Shott SR, LaRose CR, et al. Causes of persistent obstructive sleep apnea despite previous tonsillectomy and adenoidectomy in children with Down syndrome as depicted on static and dynamic cine MRI. AJR Am J Roentgenol 2004; 183:175-81.

20 Tang A, Gropler M, Duggins AL, et al. Gaps in evidence: management of pediatric obstructive sleep apnea without tonsillar hypertrophy. Laryngoscope 2016;126:758-62.

21 Lefaivre JF, Cohen SR, Burstein FD, et al. Down syndrome: identification and surgical management of obstructive sleep apnea. Plast Reconstr Surg 1997:99:629-37.

22 Cohen SR, Holmes RE, Machado L, et al. Surgical strategies in the treatment of complex obstructive sleep apnoea in children. Paediatr Respir Rev 2002;3:25-35.

23 de Moura CP, Andrade D, Cunha LM, et al. Down syndrome: otolaryngological effects of rapid maxillary expansion. J Laryngol Otol 2008;122:1318-24.

24 Kaditis AG, Alonso Alvarez ML, Boudewyns A, et al. Obstructive sleep disordered breathing in 2- to 18-year-old children: diagnosis and management. Eur Respir J 2016;47:69-94.

25 Alonso-Álvarez ML, Terán-Santos J, Navazo-Egüia Al, et al. Treatment outcomes of obstructive sleep apnoea in obese community-dwelling children: the NANOS study. Eur Respir J 2015;46:717-27. 
26 Lee $\mathrm{CH}$, Hsu WC, Chang WH, et al. Polysomnographic findings after adenotonsillectomy for obstructive sleep apnea in obese and non-obese children: a systemic review and meta-analysis. Clin Otolaryngol 2015. Published online first: Oct 5 2015. doi:10.1111/coa.12549.

27 Mitchell RB, Kelly J. Outcome of adenotonsillectomy for obstructive sleep apnea in obese and normal-weight children. Otolaryngol Head Neck Surg 2007;137:43-8.

28 Tagaya M, Nakata S, Yasuma F, et al. Relationship between adenoid size and severity of obstructive sleep apnea in preschool children. Int J Ped Otorhinolaryngol 2012;76:1827-30.
29 Levanon A, Tarasiuk A, Tal A. Sleep characteristics in children with Down syndrome. J Pediatr 1999;134:755-60.

30 Nisbet LC, Phillips NN, Hoban TF, et al. Characterization of a sleep architectural phenotype in children with Down syndrome. Sleep Breath 2015;19:1065-71.

31 Goldstein NA, Armfield DR, Kingsley LA, et al. Postoperative complications after tonsillectomy and adenoidectomy in children with Down syndrome. Arch Otolaryngol Head Neck Surg 1998;124:171-6.

32 Bower CM, Richmond D. Tonsillectomy and adenoidectomy in patients with Down syndrome. Int J Ped Otorhinolaryngol 1995;33:141-8.

\section{ARCHIVIST}

\section{How to prevent egg allergy}

Over the past couple of years, a flurry of papers has altered the way we think about food allergy in young children. Instead of avoiding a potentially allergenic food, evidence is accumulating that deliberate exposure may be beneficial. Previously, Archivist has reported on two successful peanut allergy prevention trials, STOP II (April 2014; doi:10.1136/ archdischild-2014-306139) and LEAP (May 2015; doi:10.1136/archdischild-2015-308540), and the EAT trial involving multiple food allergens which produced equivocal outcomes (Sept 2016; doi:10.1136/archdischild-2016-311602).

Now a randomised controlled trial from Japan adds to this evidence (PETIT trial. Natsume O, et al. Lancet 2016: doi.org/10.1016/S0140-6736(16)31418-0). Infants with eczema, and hence at high risk of allergy, were recruited at age 4 to 5 months, before they had eaten any egg. About 150 were randomised to receive either hen's egg or placebo. Significantly the egg was in the form of heated egg powder, and in small, increasing doses: $50 \mathrm{mg}$ a day from 6 to 9 months, and then $250 \mathrm{mg}$ a day up to $12 \mathrm{months}$. The development of true egg allergy was assessed by open oral food challenges at 12 months. Their preliminary analysis, based on only 100 of the participants, showed a striking effect: $9 \%$ of the egg exposure group had developed allergy compared to $38 \%$ of the placebo controls (risk ratio 0.22 [95\% CI 0.08-0.6; $\mathrm{p}=0.001]$ ). Importantly, there did not appear to be an increased frequency of adverse events in the egg group, suggesting that early introduction is safe. They decided to terminate the trial early on the basis of these results.

An editorial by Graham Roberts suggests that this trial was so much more definitive than previous studies because cooked rather than raw egg was used, and the 'dose' was much smaller (doi.org/10.1016/S0140-6736(16)31661-0). He regrets the early termination of this trial, because effect size may have been over-estimated, and potential adverse effects may have been missed.

Egg allergy is common, occurring in 3-4\% of infants, and as evidence accumulates, it may soon come to be regarded as preventable. However it may be a difficult task to persuade parents to give their babies a food that they consider harmful, even in tiny quantities.

Competing interests None declared.

Provenance and peer review Commissioned; internally peer reviewed.

Accepted 14 February 2017

Published Online First 2 February 2017

Arch Dis Child 2017;102:336. doi:10.1136/archdischild-2017-312881

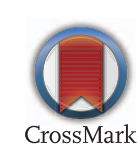




\section{ADC Outcome of adenotonsillectomy in children with Down syndrome and obstructive sleep apnoea}

Mieke Maris, Stijn Verhulst, Marek Wojciechowski, Paul Van de Heyning and An Boudewyns

Arch Dis Child 2017 102: 331-336 originally published online August 2, 2016

doi: 10.1136/archdischild-2015-310351

Updated information and services can be found at:

http://adc.bmj.com/content/102/4/331

These include:

References This article cites 31 articles, 2 of which you can access for free at: http://adc.bmj.com/content/102/4/331\#BIBL

Email alerting Receive free email alerts when new articles cite this article. Sign up in the service box at the top right corner of the online article.

\section{Notes}

To request permissions go to:

http://group.bmj.com/group/rights-licensing/permissions

To order reprints go to:

http://journals.bmj.com/cgi/reprintform

To subscribe to BMJ go to:

http://group.bmj.com/subscribe/ 